\title{
NON-PLANAR, NON-LINEAR OSCILLATIONS OF A BEAM \\ II. FREE MOTIONS
}

\author{
C.-H. Ho \\ General Electric Materials and Processes Laboratory, Schenectady, \\ New York, U.S.A. \\ AND \\ R. A. SCOTt AND J. G. Eisley \\ College of Engineering, \\ University of Michigan, Ann Arbor, Michigan 48104, U.S.A.
}

(Received 25 September 1975, and in revised form 27 February 1976)

Steady and unsteady free motions of compact beams with fixed ends are examined. It is found that in certain situations planar motions are unstable to out-of-plane perturbations and whirling motions occur. In resonant cases these whirling motions are of the beating type, whereas in non-resonant situations they have a steady-state behaviour.

\section{INTRODUCTION}

In a companion paper [1], steady-state non-linear motions of a simply supported compact beam subject to time-harmonic plane forcing were examined. Amplitude-frequency plots, and associated stability zones, were given for two force levels, for both in-plane and out-ofplane response, the major emphasis being placed on the latter, in view of the relatively little information known about them. Here, free motions of the beam are treated.

The items of prime interest are slowly changing motions. General studies of these have been given by Agrawal and Evan-Iwanowski [2], using an extended version of the KrylovBogoliubov-Mitropolski (KBM) method. It should be noted that the diversity of fields in which non-linear models arise has led to a diversity of methods of approach to the category of problem. Stanišic and Euler [3] recently presented an extension of Struble's technique. Rehfield [4] developed a perturbation method for handling both free and forced vibrations. Kabakow [5] gave a general version of the method of multiple-time-scales for multi-degreeof-freedom systems which overcame the problem of small divisors (as it frequently is called in physics works). Nayfeh, Mook and Sridhar [6] also presented general information on multipletime-scale methods. Here a KBM method is used for unsteady motions. In particular, the version employed by Gilchrist [7] will be closely followed.

\section{EQUATION OF MOTION}

The beams to be treated are symmetric, slender, and compact: that is, moments of inertia about two perpendicular axes in the cross-section (the axes of symmetry) have close values. Under these circumstances, torsional and longitudinal frequencies are considerably higher than bending ones, and the shortening effects due to torsion may be neglected. In passing, it should be noted that thin-walled sections have also been the subject of recent attention (see references [8-11]). 
When longitudinal inertia and Poisson effects are neglected, and large deformations are taken into account only through use of Green's strain measure in the longitudinal direction, the fundamental equations of motion are equations (5) and (6) of reference [1]. With the forcing functions and damping taken to be zero, these equations are

$$
\begin{aligned}
& \hat{u}_{. \mathrm{rt}}+\frac{I_{y v}}{A L^{2}}\left(\hat{u}_{. s s s s}-\beta \pi^{2} \hat{u}_{, s s}\right)-\frac{1}{2} \hat{u}_{, s s} \int_{0}^{1}\left[\left(\hat{u}_{. s}\right)^{2}+\left(\hat{v}_{. s}\right)^{2}\right] \mathrm{d} s=0, \\
& \hat{v}_{, \mathrm{rt}}+\frac{I_{y y}}{A L^{2}}\left(y \hat{v}_{. s s s}-\beta \pi^{2} \hat{v}_{. s s}\right)-\frac{1}{2} \hat{v}_{, s s} \int_{0}^{1}\left[\left(\hat{u}_{. s}\right)^{2}+\left(\hat{v}_{, s}\right)^{2}\right] \mathrm{d} s=0,
\end{aligned}
$$

where

$$
\begin{gathered}
s=z / L, \quad \tau=(t / L) \sqrt{E / \rho}, \quad \hat{u}=u / L, \quad \hat{v}=v / L, \\
\gamma-I_{x x} / I_{y y}, \quad \beta-\varepsilon_{0} / \varepsilon_{c r} \quad I_{x x}=\iint_{A} y^{2} \mathrm{~d} A, \quad I_{y y}=\iint_{A} x^{2} \mathrm{~d} A,
\end{gathered}
$$

$t$ is time, a comma stands for a partial derivative, $E$ is Young's modulus, $A$ is cross-sectional area, $\rho$ denotes density, $L$ is the length of the beam, $u$ and $v$ are the displacement components of a point on the neutral axis, in the $x, y$-directions--the symmetry directions-respectively, $\varepsilon_{0}$ is any initial strain (constant) that may be present and $\varepsilon_{c r}$ is the strain in the first buckling mode, i.e., for buckling in the $x s$-plane, $\varepsilon_{c r}=I_{y y} \pi^{2} / A L^{2}$. The displacement component in the $z$-direction, $w$, does not arise here. In arriving at equations (1) and (2) it was eliminated through use of a third equation of motion, on neglecting longitudinal inertia.

For simply supported ends the following trial solutions are adopted

$$
\begin{gathered}
\hat{u}=\sum_{m=1}^{\infty} \sin m \pi s \xi_{m}(\tau), \\
\hat{v}=\sum_{n=1}^{\infty} \sin n \pi s \eta_{n}(\tau) .
\end{gathered}
$$

Substituting equations (3) and (4) into equations (1) and (2), and using Galerkin's method gives

where

$$
\begin{aligned}
& \frac{\mathrm{d}^{2} \xi_{m}}{\mathrm{~d} \tau^{2}}+P_{x m} \xi_{m}+\frac{m^{2} \pi^{4}}{4}\left[\sum_{j=1}^{\infty}\left(\xi_{j}^{2}+\eta_{j}^{2}\right) j^{2}\right] \xi_{m}=0, \\
& \frac{\mathrm{d}^{2} \eta_{n}}{\mathrm{~d} \tau^{2}}+P_{y n} \eta_{n}+\frac{n^{2} \pi^{4}}{4}\left[\sum_{j=1}^{\infty}\left(\xi_{j}^{2}+\eta_{j}^{2}\right) j^{2}\right] \eta_{n}=0
\end{aligned}
$$

$$
P_{x m}=h \alpha^{2} m^{2} \pi^{4}\left(m^{2}+\beta\right), \quad P_{y n}=h \alpha^{2} n^{2} \pi^{4}\left(\gamma n^{2}+\beta\right), \quad h=I_{y y} / A d^{2}, \quad \alpha=d / L,
$$

and $d$ is the depth of the beam (the maximum dimension in the $x s$-plane).

The possibility of multi-mode interactions is exhibited by equations (5) and (6). Here the only item that will be pursued is one that is felt to be of common occurrence, namely, one mode in each plane dominating. With $m$ and $n$ denoting the dominant modes in the $x s-$, and $y s$-plane, respectively, equations (5) and (6) reduce to

$$
\begin{gathered}
\mathrm{d}^{2} \xi_{m} / \mathrm{d} \tau^{2}+P_{x m} \xi_{m}+\left(m^{2} \pi^{4} / 4\right)\left(m^{2} \xi_{m}^{2}+n^{2} \eta_{n}^{2}\right) \xi_{m}=0, \\
\mathrm{~d}^{2} \eta_{n} / \mathrm{d} \tau^{2}+P_{y n} \eta_{n}+\left(n^{2} \pi^{4} / 4\right)\left(m^{2} \xi_{m}^{2}+n^{2} \eta_{n}^{2}\right) \eta_{n}=0 .
\end{gathered}
$$

Steady-state, harmonic motions in the $x s$-plane can be investigated by assuming

$$
\xi_{m}=A_{x m} \cos \omega \tau, \quad \eta_{n}=0,
$$

where $A_{x m}$ is an amplitude, and $\omega$ denotes frequency. Substituting equations (9) into equation (7) generates a Duffing equation, which is handled here by the method of harmonic balance. 
The frequency-amplitude relation is

$$
A_{x m}\left(P_{x m}-\omega^{2}\right)+\left(3 m^{4} \pi^{4} / 16\right) A_{x m}^{3}=0 .
$$

The existence of motions satisfying equation (10) is problematical, until their stability when subjected to perturbations has been investigated. Two types of perturbation must be considered, namely, ones in the $x$ s-plane ( $\equiv$ in-plane), and ones in the $y_{s}$-plane ( $\equiv$ out-ofplane). Upon letting

$$
\begin{gathered}
\xi_{m} \rightarrow \xi_{m}+\xi_{m}^{\prime}, \quad m=1,2, \ldots, \\
\eta_{n} \rightarrow \eta_{n}+\eta_{n}^{\prime}, \quad n=1,2, \ldots,
\end{gathered}
$$

where $\xi_{m}$ and $\eta_{n}$ as are given by equations (9), it can be shown that equations (5) and (6) yield, after considerable algebra,

$$
\begin{array}{ll}
\mathrm{d}^{2} \xi_{j}^{\prime} / \mathrm{d} z^{2}+\left(\Delta_{x j}+\varepsilon_{x j} \cos z\right) \xi_{j}^{\prime}=0, & j=1,2, \ldots, \\
\mathrm{d}^{2} \eta_{j}^{\prime} / \mathrm{d} z^{2}+\left(\Delta_{y j}+\varepsilon_{y j} \cos z\right) \eta_{j}^{\prime}=0, & j=1,2, \ldots,
\end{array}
$$

where now $z=2 \omega \tau$ and

$$
\begin{gathered}
\Delta_{x j}=P_{x j} / 4 P_{x m}+\left[1-3 m^{2} P_{x j} / 2\left(1+2 \delta_{j m}\right) j^{2} P_{x m}\right] \varepsilon_{x j}, \\
\varepsilon_{x j}=\left\{\left(1+2 \delta_{j m}\right) m^{2} j^{2} \pi^{4} A_{x m}^{2} /\left[8 P_{x j}+\left(1+2 \delta_{j m}\right) m^{2} j^{2} \pi^{4} A_{x m}^{2}\right]\right\} \Delta_{x j}, \\
\Delta_{y j}=P_{y j} / 4 P_{x m}+\left(1-3 m^{2} P_{y j} / 2 j^{2} P_{x m}\right) \varepsilon_{y j}, \\
\varepsilon_{y j}=\left[m^{2} j^{2} \pi^{4} A_{x m}^{2} /\left(8 P_{y j}+m^{2} j^{2} \pi^{4} A_{x m}^{2}\right)\right] \Delta_{y j},
\end{gathered}
$$

$\delta$ denoting the Kronecker delta.

Equations (13) and (14) are Mathieu equations and so exact information regarding stability is available. However an approximate scheme will be used here, since the ensuing results are better suited for comparisons with the approximate response information given by equation (10). The scheme, which is described fully in reference [1], hinges around expansions in powers of characteristic exponents. It yields, for the first Mathieu instability zone (the only one considered in the sequel),

$$
\Delta_{r j}=\frac{1}{4} \pm \frac{1}{2} \varepsilon_{r j}, \ldots,
$$

where $r$ stands for either $x$ or $y$. Equation (19) corresponds to straight lines in the $\varepsilon \Delta$-plane, and these are denoted by AC and AE in Figure 1. Shaded areas in that diagram correspond to stable motions. As has been shown by Stoker [12] for the Duffing equation, points on the boundary between stability and instability regions correspond to orbitally stable motions:

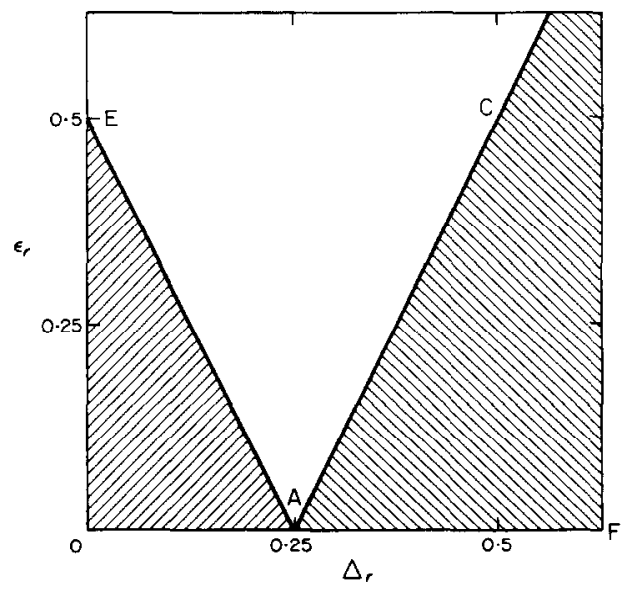

Figure 1. Approximate Mathieu stability boundaries. 
that is, if such a motion is subject to a small disturbance, the subsequent motion differs only slightly in amplitude and frequency from the original.

Equations (15) and (16) - and (17) and (18)-- are straight lines in the $\varepsilon \Delta$-plane. The stability associated with points in the $A_{x m} \omega$-plane can be established from the location of the point of intersection of these straight lines, and the results are as follows. Consider first the case $j=m$. The modes $\xi_{m}$ are always orbitally stable: that is, a perturbation $\xi_{m}^{\prime}$ just leads to a $\xi_{m}$ motion with a different amplitude. When $m=1, j=2,3, \ldots$, it is found that $\xi_{1}$ motions do not excite $\xi_{2}, \xi_{3}, \ldots$, motions, in that $\xi_{2}^{\prime}, \xi_{3}^{\prime}, \ldots$, do not grow. Similarly, it is found that $\xi_{2}$ motions $(m=2)$ do not excite the $\xi_{4}, \xi_{5}, \ldots$, modes, nor the $\xi_{1}$ mode. In contrast, $\xi_{3}$ motions are generated. The pattern procedes, with the possibility of $\xi_{4}, \xi_{5}$ modes growing when $m=3$. Identical results can be argued for motions and perturbation in the $y s$-plane. A point of interest is that these conclusions do not depend on the value of $\beta$ (provided it is less than one): i.e., they are independent of the initial strain state.

Out-of-plane perturbations will now be considered. In this case the results do depend on the value of $\beta$, which is taken here to be zero. Consider first the situation where $m=j=1$. From equations (17) and (18) and the stability chart, it can be shown that $\eta_{1}$ perturbations grow only for $\gamma>1$. For $\gamma<1$, the motions in the $x s$-plane are stable to out-of-plane perturbations. For $\gamma=1$, orbital stability prevails. For out-of-plane modes other than the first, i.e., $j=2,3, \ldots$, it is interesting that whirling can occur for $\gamma<1$, provided $\gamma>1 / j^{2}$. Consider now the second mode in the $x s$-plane: i.e., $m=2 . \eta_{1}^{\prime}$ perturbations grow only if $\gamma>16$. $\eta_{2}^{\prime}$ motions may grow for $\gamma>1$, but not for $\gamma<1$, and so on. The above results could have been obtained, for the undamped case, on setting the forcing functions in reference [1] equal to zero.

The possibility of unsteady, free non-planar motions (motions that were not treated in reference [1]) existing will now be explored. Upon making the scale changes

$$
\begin{aligned}
\xi_{m} & =\sqrt{\varepsilon} x_{m}, \\
\eta_{n} & =\sqrt{\varepsilon} y_{n},
\end{aligned}
$$

where $\varepsilon$ is regarded as being small enough to consider the products in equations (20) and (21) as small, equations (7) and (8) become

$$
\begin{gathered}
\mathrm{d}^{2} x_{m} / \mathrm{d} \tau^{2}+P_{x m} x_{m}+\varepsilon\left(m^{2} \pi^{4} / 4\right)\left(m^{2} x_{m}^{2}+n^{2} y_{n}^{2}\right) x_{m}=0, \quad m=1,2, \ldots, \\
\mathrm{d}^{2} y_{n} / \mathrm{d} \tau^{2}+P_{y n} y_{n}+\varepsilon\left(n^{2} \pi^{4} / 4\right)\left(m^{2} x_{m}^{2}+n^{2} y_{n}^{2}\right) y_{n}=0, \quad n=1,2, \ldots
\end{gathered}
$$

Equations similar to equations (22) and (23) have been examined, for example, by Gilchrist [7], using a KBM method. His method of analysis will be closely pursued here.

In approaching the weakly coupled, non-linear, ordinary differential equations (22) and (23) the mathematical tack adopted by many schemes depends on whether a condition of internal resonance exists or not. By internal resonance is meant a circumstance in which certain frequencies, or combination of frequencies, generated in the scheme by the non-linear terms in the differential equations, equal linear natural frequencies. The phrase " $r$ th order internal resonance" is common. It signifies that the term(s) in question arise at the $r$ th level of the approximate scheme. For certain systems, this depends on the linear frequency ratios of the various modes. For instance, for the case at hand, if

$$
\sqrt{P_{x m}}=3 \sqrt{P_{y n}}
$$

then the scheme, which involves expansion in powers of $\varepsilon$, would predict resonance between these two modes when terms of order $\varepsilon^{3}$ are taken into account. It would also occur if equation (24) were only satisfied approximately. Generally speaking, such resonances take place when the following equations are satisfied either exactly or approximately:

$$
P_{x m}=(\omega / i)^{2}+\varepsilon \Gamma_{x m}
$$




$$
P_{y n}=(\omega / j)^{2}+\varepsilon \Gamma_{y n}
$$

where $\omega$ is related to the frequency of free-vibrations, as can be seen on setting $\varepsilon$ equal to zero. Substituting equations (25) and (26) into equations (22) and (23) gives

$$
\begin{aligned}
& \frac{\mathrm{d}^{2} x_{m}}{\mathrm{~d} \tau^{2}}+\left(\frac{\omega}{i}\right)^{2} x_{m}+\varepsilon \frac{m^{2} \pi^{4}}{4}\left(m^{2} x_{m}^{2}+n^{2} y_{n}^{2}+\frac{4 \Gamma_{x m}}{m^{2} \pi^{4}}\right) x_{m}=0, \\
& \frac{\mathrm{d}^{2} y_{n}}{\mathrm{~d} \tau^{2}}+\left(\frac{\omega}{j}\right)^{2} y_{n}+\varepsilon \frac{n^{2} \pi^{4}}{4}\left(m^{2} x_{m}^{2}+n^{2} y_{n}^{2}+\frac{4 \Gamma_{y n}}{n^{2} \pi^{4}}\right) y_{n}=0 .
\end{aligned}
$$

Solutions to equations (27) and (28) are sought in the form

$$
\begin{aligned}
& x_{m}=a_{m} \cos \left(\frac{\omega}{i} \tau+\theta_{m}\right)+\varepsilon g_{m}^{(1)}\left(a_{m}, b_{n}, \theta_{m}, \phi_{n}, \tau\right)+\varepsilon^{2} g_{m}^{(2)}+\ldots, \\
& y_{n}=b_{n} \sin \left(\frac{\omega}{j} \tau+\phi_{n}\right)+\varepsilon h_{n}^{(1)}\left(a_{m}, b_{n}, \theta_{m}, \phi_{n}, \tau\right)+\varepsilon^{2} h_{n}^{(2)}+\ldots,
\end{aligned}
$$

where $g_{m}^{(s)}$ and $h_{n}^{(s)}, s=1,2, \ldots$, are periodic functions of $\theta_{m}$ and $\phi_{n}$, and $a_{m}, b_{n}, \theta_{m}$, and $\phi_{n}$ are determined from

$$
\begin{aligned}
& \mathrm{d} a_{m} / \mathrm{d} \tau=\varepsilon A_{m}^{(1)}\left(a_{m}, b_{n}, \theta_{m}, \phi_{n}\right)+\varepsilon^{2} A_{m}^{(2)}\left(a_{m}, b_{n}, \theta_{m}, \phi_{n}\right)+\ldots, \\
& \mathrm{d} b_{n} / \mathrm{d} \tau=\varepsilon B_{n}^{(1)}\left(a_{m}, b_{n}, \theta_{m}, \phi_{n}\right)+\varepsilon^{2} B_{n}^{(2)}\left(a_{m}, b_{n}, \theta_{m}, \phi_{n}\right)+\ldots, \\
& \mathrm{d} \theta_{m} / \mathrm{d} \tau=\varepsilon C_{m}^{(1)}\left(a_{m}, b_{n}, \theta_{m}, \phi_{n}\right)+\varepsilon^{2} C_{m}^{(2)}\left(a_{m}, b_{n}, \theta_{m}, \phi_{n}\right)+\ldots, \\
& \mathrm{d} \phi_{n} / \mathrm{d} \tau=\varepsilon D_{n}^{(1)}\left(a_{m}, b_{n}, \theta_{m}, \phi_{n}\right)+\varepsilon^{2} D_{n}^{(2)}\left(a_{m}, b_{n}, \theta_{m}, \phi_{n}\right)+\ldots,
\end{aligned}
$$

where $A_{m}^{(1)}$ through $D_{n}^{(2)}$ are periodic in $\theta_{m}$ and $\phi_{n}$. Substituting equations (31) through (34) into equations (27) and (28), and expanding the non-linear terms in these last equations, gives, on specializing to the case of first order internal resonance, that is, $i=j=1$,

$$
\begin{aligned}
\partial^{2} g_{m}^{(1)} / \partial \tau^{2}+\omega^{2} g_{m}^{(1)}=[ & \left.2 \omega A_{m}^{(1)}-\left(q_{x m} / 4\right) n^{2} a_{m} b_{n}^{2} \sin \left(2 \phi_{n}-2 \theta_{m}\right)\right] \sin \left(\omega \tau+\theta_{m}\right)+ \\
& +\left\{2 a_{m} \omega C_{m}^{(1)}-\Gamma_{x m} a_{m}-\left(q_{x m} / 4\right)\left[3 m^{2} a_{m}^{3}+n^{2} a_{m} b_{n}^{2}(2-\right.\right. \\
& \left.\left.\left.-\cos \left(2 \phi_{n}-2 \theta_{m}\right)\right)\right]\right\} \cos \left(\omega \tau+\theta_{m}\right)-\left(q_{x m} / 4\right) m^{2} a_{m}^{3} \cos \left(3 \omega \tau+3 \theta_{m}\right)+ \\
& +\left(q_{x m} / 4\right) n^{2} a_{m} b_{n}^{2} \cos \left(3 \omega \tau+\theta_{m}+2 \phi_{n}\right), \\
\partial^{2} h_{n}^{(1)} / \partial \tau^{2}+\omega^{2} h_{n}^{(1)}= & \left.-2 \omega B_{n}^{(1)}-\left(q_{y n} / 4\right) m^{2} a_{m}^{2} b_{n} \sin \left(2 \phi_{n}-2 \theta_{m}\right)\right] \cos \left(\omega \tau+\phi_{n}\right)+ \\
& +\left\{2 b_{n} \omega D_{n}^{(1)}-\Gamma_{y n} b_{n}-\left(q_{y n} / 4\right)\left[3 n^{2} b_{n}^{3}+m^{2} a_{m}^{2} b_{n}(2-\right.\right. \\
& \left.\left.\left.-\cos \left(2 \phi_{n}-2 \theta_{m}\right)\right)\right]\right\} \sin \left(\omega \tau+\phi_{n}\right)+\left(q_{y n} / 4\right) n^{2} b_{n}^{3} \sin \left(3 \omega \tau+3 \phi_{n}\right)- \\
& -\left(q_{y n} / 4\right) m^{2} a_{m}^{2} b_{n} \sin \left(3 \omega \tau+2 \theta_{m}+\phi_{n}\right),
\end{aligned}
$$

where $q_{x m}=m^{2} \pi^{4} / 4$ and $q_{y n}=n^{2} \pi^{4} / 4$.

To suppress secularities in equations (35) and (36), the coefficients of harmonic functions with frequency $\omega$ must be set to zero. In this way $A_{m}^{(1)}, B_{n}^{(1)}, C_{m}^{(1)}$ and $D_{n}^{(1)}$ can be determined. Substituting these values into equations (29) through (34) yields

$$
\begin{gathered}
x_{m}=a_{m} \cos \left(\omega \tau+\theta_{m}\right)+\varepsilon g_{m}^{(1)}, \\
y_{n}=b_{n} \sin \left(\omega \tau+\phi_{n}\right)+\varepsilon h_{n}^{(1)}, \\
\mathrm{d} a_{m} / \mathrm{d} \tau=\varepsilon A_{m}^{(1)}=(\varepsilon / 8 \omega) q_{x m} n^{2} b_{n}^{2} a_{m} \sin \left(2 \phi_{n}-2 \theta_{m}\right), \\
\mathrm{d} b_{n} / \mathrm{d} \tau=\varepsilon B_{n}^{(1)}=-(\varepsilon / 8 \omega) q_{y n} m^{2} a_{m}^{2} b_{n} \sin \left(2 \phi_{n}-2 \theta_{m}\right), \\
\mathrm{d} \theta_{m} / \mathrm{d} \tau-\varepsilon C_{m}^{(1)}-(\varepsilon / 2 \omega)\left\{\Gamma_{x m}+(3 / 4) q_{x m} m^{2} a_{m}^{2}+(1 / 4) q_{x m} n^{2} b_{n}^{2}\left[2-\cos \left(2 \phi_{n}-2 \theta_{m}\right)\right],\right. \\
\mathrm{d} \phi_{n} / \mathrm{d} \tau=\varepsilon D_{n}^{(1)}=(\varepsilon / 2 \omega)\left\{\Gamma_{y n}+(3 / 4) q_{y n} n^{2} b_{n}^{2}+(1 / 4) q_{y n} m^{2} a_{m}^{2}\left[2-\cos \left(2 \phi_{n}-2 \theta_{m}\right)\right]\right\} .
\end{gathered}
$$


Gilchrist [7] succeeded in partially integrating equations similar to (39) through (42). Dividing equation (39) by (40) and integrating gives

$$
a_{m}^{2}+b_{n}^{2}=C,
$$

where $C$ is a constant of integration. Dividing equation (41) by (42), making the variable changes

and integrating, yields

$$
\begin{gathered}
a_{m}=\sqrt{C} \cos \chi, \\
b_{n}=\sqrt{C} \sin \chi, \\
\Phi=\phi_{n}-\theta_{m},
\end{gathered}
$$

where

$$
-2 \psi \cos 2 \chi+\sin ^{2} 2 \chi(f+\cos 2 \Phi)=C_{0}
$$

$$
\begin{gathered}
\psi=16\left(\Gamma_{x m}-\Gamma_{y n}\right) / C n^{2} m^{2} \pi^{4}-(3 / 2)\left[\left(n^{2} / m^{2}\right)-\left(m^{2} / n^{2}\right)\right], \\
f=(3 / 2)\left[\left(n^{2} / m^{2}\right)+\left(m^{2} / n^{2}\right)\right]-2
\end{gathered}
$$

and $C_{0}$ is a constant of integration.

In general it is difficult to express the motion explicitly in terms of time. However, it is possible to do this in the special case $m=n, \gamma=1$ (Anand [13] obtained similar results). One gets

$$
\begin{gathered}
a_{m}^{2}+b_{m}^{2}=c_{1}^{2}, \\
a_{m}^{2} b_{m}^{2}=c_{2}^{2} \sec ^{2}\left(\phi_{m}-\theta_{m}\right), \\
a_{m}^{2}=\left(c_{1}^{2} / 2\right)+\sqrt{\left(c_{1}^{2} / 2\right)^{2}-c_{2}^{2}} \sin \left[\left(\varepsilon m^{2} q_{x m} c_{2} / 2 \omega\right) \tau+c_{3}\right], \\
b_{m}^{2}=\left(c_{1}^{2} / 2\right)-\sqrt{\left(c_{1}^{2} / 2\right)^{2}-c_{2}^{2}} \sin \left[\left(\varepsilon m^{2} q_{x m} c_{2} / 2 \omega\right) \tau+c_{4}\right], \\
\theta_{m}=\frac{3 \varepsilon}{8 \omega} q_{x m} m^{2} c_{1}^{2} \tau-\tan ^{-1}\left\{\frac{c_{1}^{2} \tan \left[\left(\varepsilon m^{2} q_{x m} c_{2} / 4 \omega\right) \tau+c_{3} / 2\right]+\sqrt{c_{1}^{4}-4 c_{2}^{2}}}{2 c_{2}}\right\}+c_{5}, \\
\phi_{m}=\frac{3 \varepsilon}{8 \omega} q_{y m} m^{2} c_{1}^{2} \tau-\tan ^{-1}\left(\frac{c_{1}^{2} \tan \left[\left(\varepsilon m^{2} q_{x m} c_{2} / 4 \omega\right) \tau+c_{4} / 2\right]-\sqrt{c_{1}^{4}-4 c_{2}^{2}}}{2 c_{2}}\right\}+c_{6},
\end{gathered}
$$

where $c_{1}$ through $c_{6}$ are constants of integration (note that only four of the six are independent) to be determined from initial conditions.

Equations (43) through (55) show that unsteady motions do indeed exist, the amplitudes and phases in both the $x s$ and $y s$ planes being slowly varying functions of time. In the special case $m=n, P_{x m}=P_{y n}$, following Anand [13], it can be shown that a particle trajectory is a rotating ellipse, with the axis rotating each revolution

$$
\Delta \theta=\left(\varepsilon q_{x m} \pi / 2 \sqrt{P_{x m}}\right)\left[r^{2}(0)+r^{2}(0) \dot{\theta}^{2}(0) / P_{x m}+\dot{r}^{2}(0) / P_{x m}\right],
$$

where $r$ and $\theta$ are polar co-ordinates. It should be noted that the various types of beating motions that can arise can be analyzed with the aid of a $\Phi \chi$-phase plane (see equations (44), (45) and (46)). The reader is referred to Gilchrist's paper [7] for more detail.

To conclude, some comments on non-resonant motions will be made. To investigate these, one takes

$$
\begin{aligned}
& x_{m}=a_{m} \cos \left(\sqrt{P_{x m}} \tau+\theta_{m}\right)+\varepsilon g_{m}^{(1)}\left(a_{m}, b_{n}, \theta_{m}, \phi_{n}, \tau\right)+\varepsilon^{2}+\ldots, \\
& y_{n}=b_{n} \sin \left(\sqrt{P_{y n}} \tau+\theta_{n}\right)+\varepsilon h_{n}^{(1)}\left(a_{m}, b_{n}, \theta_{m}, \phi_{n}, \tau\right)+\varepsilon^{2}+\ldots
\end{aligned}
$$


The previously described procedure now yields

$$
\begin{aligned}
& \mathrm{d} a_{m} / \mathrm{d} \tau=\varepsilon A_{m}^{(1)}=0, \quad \mathrm{~d} b_{n} / \mathrm{d} \tau=\varepsilon B_{n}^{(1)}=0, \\
& \mathrm{~d} \theta_{m} / \mathrm{d} \tau=\varepsilon C_{m}^{(1)}=\varepsilon\left[q_{x m} / 8\left(P_{x m}\right)^{\ddagger}\right]\left(3 m^{2} a_{m}^{2}+2 n^{2} b_{n}^{2}\right), \\
& \mathrm{d} \phi_{n} / \mathrm{d} \tau=\varepsilon D_{n}^{(1)}=\varepsilon\left[q_{y n} / 8\left(P_{y n}\right)^{\frac{1}{t}}\right]\left(3 n^{2} b_{n}^{2}+2 m^{2} a_{m}^{2}\right)
\end{aligned}
$$

Hence, since the amplitudes are now constant, no beating motions occur, in strong contrast to the resonant case.

\section{REFERENCES}

1. C.-H. Ho, R. A. ScotT and J. G. EISLEY 1975 International Journal of Non-Linear Mechanics 9, 113-127. Non-planar, non-linear oscillations of a beam-I. Forced motions.

2. B. N. Agrawal and R. M. Evan-Iwanowski 1973 American Institute of Aeronautics and Astronautics Journal 11, 907-1001. Resonances in non-stationary, non-linear multi-degree-offreedom systems.

3. M. M. StANIŠIC and J. A. EuLER 1973 International Journal of Non-Linear Mechanics 8, 523-537. General perturbational solution of a harmonically forced non-linear oscillator equation.

4. L. W. ReHFIELD 1974 American Institute of Aeronautics and Astronautics Journal 12, 388-390. Large amplitude forced vibrations of elastic structures.

5. H. KaBAKow 1972 International Journal of Non-Linear Mechanics 7, 125-137. A perturbation procedure for weakly coupled oscillators.

6. A. H. NAYFeh, D. T. MoOK and S. SRIDHAR 1974 Journal of the Acoustical Society of America 55, 281-291. Non-linear analysis of the forced response of structural elements.

7. A. O. Gilchrist 1961 International Journal of Mechanical Science 3, 286-311. The free oscillations of conservative quasi-linear systems with two degrees of freedom.

8. C. H. POPELAR 1972 Proceedings of the American. Society of Civil Engineers, Journal of the Engineering Mechanics Division 98, 657-663. Dynamic stability of thin-walled column.

9. A. A. Ghobarah and W. K. Tso 1972 Journal of Applied Mechanics 39, 201-214. Parametric stability of thin-walled beams of open section.

10. J. Dugundu and V. Mukhabadhyay 1973 Journal of Applied Mechanics 40, 693-701. Lateral bending-torsion vibrations of a thin beam under parametric excitation.

11. S. Ali Hasan and A. D. S. BarR 1974 Journal of Sound and Vibration 32, 25-47. Non-linear and parametric vibration of thin-walled beams of equal angle-section.

12. J. J. STOKer 1950 Non-linear Vibrations. New York: Interscience Publishers, Inc.

13. G. V. ANAND 1969 Journal of the Acoustical Society of America 45, 1089-1096. Stability of nonlinear oscillations of stretched strings. 